\title{
Indole-3-acetic acid (IAA) production by Arthrobacter species isolated from Azolla
}

\author{
C. Forni, ${ }^{1 *}$ J. Riov $^{2}$ M. Grilli Caiola ${ }^{1}$ and E. Tel-OR ${ }^{3}$ \\ ${ }^{1}$ Dipartimento di Biologia, II Universita di Roma, Via Emanuele Carnevale, 00173 Roma, Italy \\ ${ }^{2}$ Department of Horticulture and ${ }^{3}$ Department of Agricultural Botany, Hebrew University of Jerusalem, Rehovot, Israel
}

(Received 26 June 1991; revised 24 September 1991; accepted 8 November 1991)

\begin{abstract}
Arthrobacter species, isolated from the leaf cavities and the microsporocarps of the aquatic fern species $A$ zolla pinnata and Azolla filiculoides, produced indole-3-acetic acid (IAA) in culture when the precursor tryptophan was added to the medium. No IAA production was detected in the absence of tryptophan. Maximum IAA formation was obtained in the first $\mathbf{2} \mathrm{d}$ of incubation. Part of the tryptophan was transformed to $\mathrm{Na}$-acetyl-L-tryptophan.
\end{abstract}

\section{Introduction}

Azolla Lam. is a genus of heterosporous aquatic fern which contains within the leaf cavities the $\mathrm{N}_{2}$-fixing cyanobacterium Anabaena azollae Strasb. The host Azolla synthesizes and provides photosynthates essential for the nitrogenase activity of the cyanobiont, while Anabaena azollae supplies fixed nitrogen to the fern (Peters \& Meeks, 1989).

The leaf cavities of the fern also contain heterotrophic eubacteria (Grilli Caiola et al., 1988; Nierzwicki-Bauer \& Aulfinger, 1990; Plazinski et al., 1990) including high numbers of the genus Arthrobacter Conn and Dimmick (Forni et al., 1989; Petro \& Gates, 1987; Wallace \& Gates, 1986). Arthrobacter spp. are ubiquitous in the fern being present not only in the leaf cavities of the Azolla species studied, but also in the sporocarps of Azolla filiculoides (Forni et al., 1990). Therefore it may be possible that bacteria are a third component of the symbiosis (Forni et al., 1989; Petro \& Gates, 1987). It is not known if the bacteria are physiologically significant for the plant or what their role is in the association.

It is known that several micro-organisms exert a marked influence on the growth of plants by producing plant growth regulators. Preliminary data have shown that some of the bacterial strains isolated from Azolla produce the plant hormone indole-3-acetic acid (IAA) (Forni \& Grilli Caiola, 1988). It is well-established that IAA regulates plant growth. Moreover, it has been suggested that IAA plays a role in bacteria-plant

*Author for correspondence. Tel: 6 72594345; fax: 62023500.

Abbreviation: IAA, indole-3-acetic acid. associations in the rhizosphere, such as Azospirillum and grass roots (Harari et al., 1989) and in symbiosis, such as Rhizobium-legumes (Prinsen et al., 1991). This work was undertaken to determine if IAA is produced by Arthrobacter species isolated from the leaf cavities and sporocarps of Azolla.

\section{Methods}

Bacterial strains. Arthrobacter globiformis Conn \& Dimmick (strain A3) (Forni et al., 1989) and Arthrobacter nicotianae GiovannozziSermanni (strain MC8) (Forni et al., 1990) were isolated respectively from the leaf cavity and from the microsporocarp of Azolla filiculoides Lam. Arthrobacter crystallopoietes Ensign and Rittenberg (strain E1) (Forni et al., 1989) was isolated from the leaf cavity of Azolla pinnata Brown. All the strains belong to the collection of the Department of Biology, II University of Rome.

Growth of bacteria. The purity of the strains utilized for the study was routinely checked by streaking on TRN (Forni et al., 1989) agar plates. Axenic cultures were grown in TRN medium for $4 \mathrm{~d}$. The cells were harvested, washed three times with mineral medium M9 (Forni et al., 1989) and inoculated in $250 \mathrm{ml}$ flasks containing medium $\mathrm{M} 9$ supplemented with $10 \mathrm{~mm}$-sucrose and different concentrations of L-tryptophan (Sigma). The cultures were incubated at $30^{\circ} \mathrm{C}$ and 150 r.p.m. (New Brunswick orbital shaker). Samples for IAA determinations were taken every day for the first $\mathbf{4} \mathrm{d}$ of the experiments. The data are means of three replicates.

Protein was determined by the Lowry method using bovine serum albumin as a standard.

IAA extraction and determination. The supernatant fraction, obtained after cell centrifugation for $20 \mathrm{~min}$ at $5500 \mathrm{~g}$, was adjusted to $\mathrm{pH} 2 \cdot 5$. The solution was applied to a C-18 Sep-Pak cartridge (Sagee et al., 1986). The cartridge was washed with distilled water and IAA was eluted with methanol containing $10 \mu \mathrm{g}$ butylated hydroxytoluene $\mathrm{ml}^{-1}$. The methanol solution containing IAA was evaporated under vacuum 


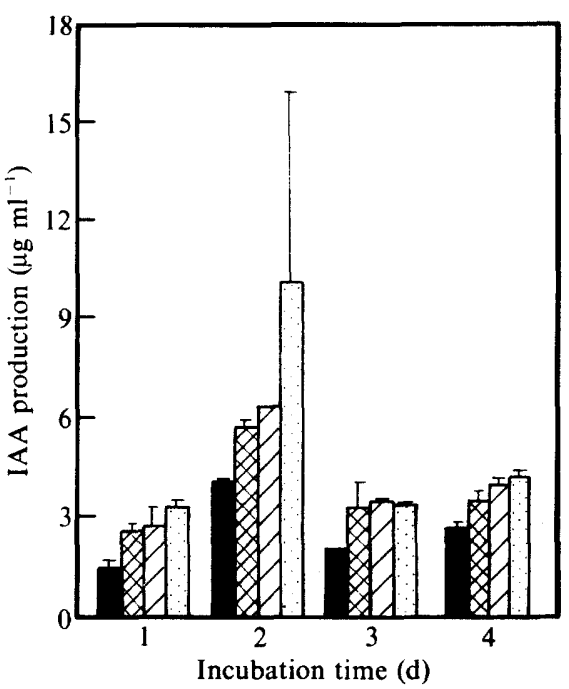

Fig. 1

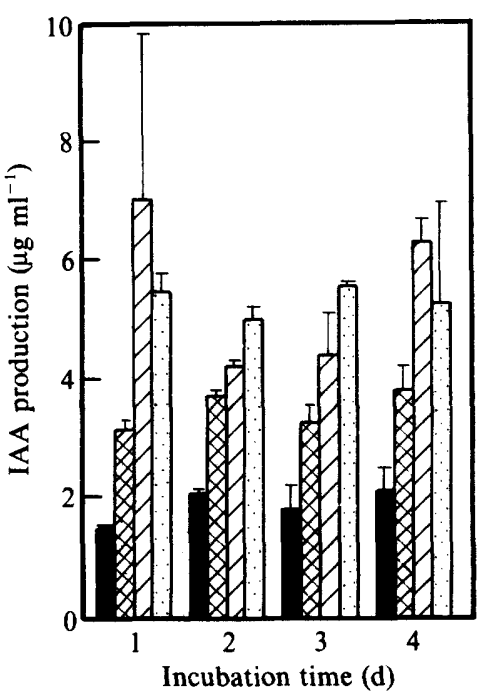

Fig. 2

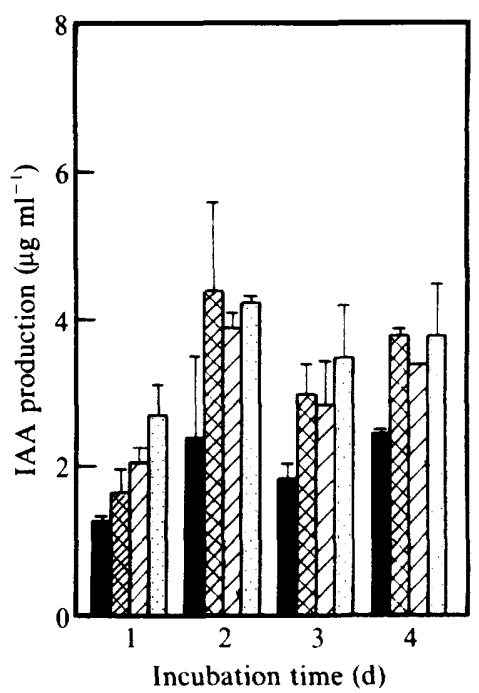

Fig. 3

Fig. 1. Production of IAA in M9 medium by a strain of Arthrobacter globiformis, isolated from the leaf cavity of Azolla filiculoides. The tryptophan concentration $\left(\mu \mathrm{g} \mathrm{ml}^{-1}\right)$ in $\mathrm{M9}$ medium was as follows: $\square, 100 ;$, $200 ; \square, 400 ; 0,600$.

Fig. 2. Production of IAA in M9 medium by a strain of Arthrobacter crystallopoietes, isolated from leaf cavity of Azolla pinnata. Symbols, as Fig. 1.

Fig. 3. Production of IAA in M9 medium by a strain of Arthrobacter nicotianae, isolated from the microsporocarp of Azolla filiculoides. Symbols, as Fig. 1.

and IAA was dissolved in $200 \mu$ methanol. IAA was determined using Salkowski reagent (Ehmann, 1977).

Isolation of tryptophan metabolites. Cultures were centrifuged as above and the $\mathrm{pH}$ was adjusted to $2 \cdot 5$. Tryptophan metabolites were extracted with two equal volumes of ethyl acetate. The ethyl acetate was evaporated to dryness and the metabolites were methylated with diazomethane (Schlenk \& Gellerman, 196C) for gas chromatographymass spectrometry (GC-MS) analysis.

$G C-M S$. This was performed on a Finigan MAT 4600 mass spectrometer. Capillary GC was carried out on a $15 \mathrm{~m} \times 0.32 \mathrm{~mm}$ i.d. DB-5 column (J \& W Scientific) with a film thickness of $0.25 \mu \mathrm{m}$. The helium flow rate was $1.2 \mathrm{ml} \mathrm{min}^{-1}$ and the injector temperature was $250{ }^{\circ} \mathrm{C}$. A temperature program of $120-220^{\circ} \mathrm{C}$ at $3{ }^{\circ} \mathrm{C} \mathrm{min}-1$ was started upon injection. Mass spectra were recorded at an ionizing voltage of $70 \mathrm{eV}$ with a source temperature of $270^{\circ} \mathrm{C}$.

\section{Results}

\section{IAA production in culture}

The growth rate of the bacteria in M9 medium supplemented with tryptophan was very low. The cell concentrations $\left(10^{7}\right.$ cells $\left.\mathrm{ml}^{-1}\right)$ and protein content did not increase during the $4 \mathrm{~d}$ of the experiments and fell within the range $1-3 \mathrm{mg}$ protein $\mathrm{ml}^{-1}$. Nevertheless, all the Arthrobacter species isolated from Azolla were able to produce IAA using tryptophan as a precursor (Figs 1, 2 and 3) since no IAA was produced in controls without tryptophan (data not shown).

The maximum production of IAA by $A$. globiformis (i.e. $10 \cdot 1 \mu \mathrm{g} \mathrm{IAA} \mathrm{ml} \mathrm{I}^{-1}$ in M9 containing $600 \mu \mathrm{g}$ tryptophan $\mathrm{ml}^{-1}$ ) and $A$. nicotianae (i.e $4.4 \mu \mathrm{g} \mathrm{IAA} \mathrm{ml}^{-1}$ in M9 containing $400 \mu \mathrm{g}$ tryptophan $\mathrm{ml}^{-1}$ ) was obtained in the second day of the experiment (Figs 1 and 3), while $A$. crystallopoietes (Fig. 2) produced high levels of IAA during the $4 \mathrm{~d}$ of incubation. The IAA content in the growth medium varied according to the concentration of tryptophan added to the culture, generally being higher at 400 and $600 \mu \mathrm{g}$ tryptophan $\mathrm{ml}^{-1}$ (Figs 1,2 and 3). IAA production by the bacteria (from 0.5 to $2 \mu \mathrm{g} \mathrm{ml}^{-1}$ ) was also detected at the lower tryptophan concentrations of $25 \mu \mathrm{g}$ and $50 \mu \mathrm{g} \mathrm{ml}^{-1}$.

IAA production was similar in TRN and M9 media containing $600 \mu \mathrm{g}$ tryptophan $\mathrm{ml}^{-1}$ : after $3 \mathrm{~d}$ growth in TRN $A$. nicotianae produced $3.3 \mu \mathrm{g}$ IAA ml ${ }^{-1}$ compared to $3.5 \mu \mathrm{g} \mathrm{IAA} \mathrm{ml}^{-1}$ in M9, while $A$. globiformis produced 3.3 and $4.4 \mu \mathrm{g} \mathrm{IAA} \mathrm{ml}^{-1}$ in TRN and in M9 respectively.

\section{Identification of tryptophan metabolites.}

Two major tryptophan metabolites were identified by GC-MS in all cultures analysed: IAA (Fig. 4) and N $\alpha$ acetyl-L-tryptophan (Fig. 5). IAA was the major meta- 


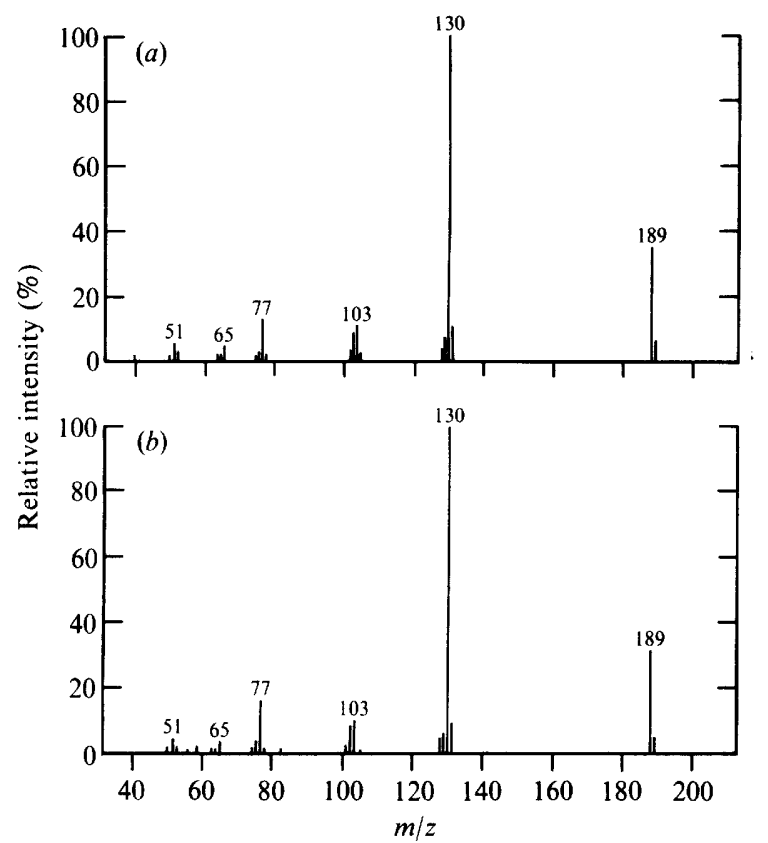

Fig. 4

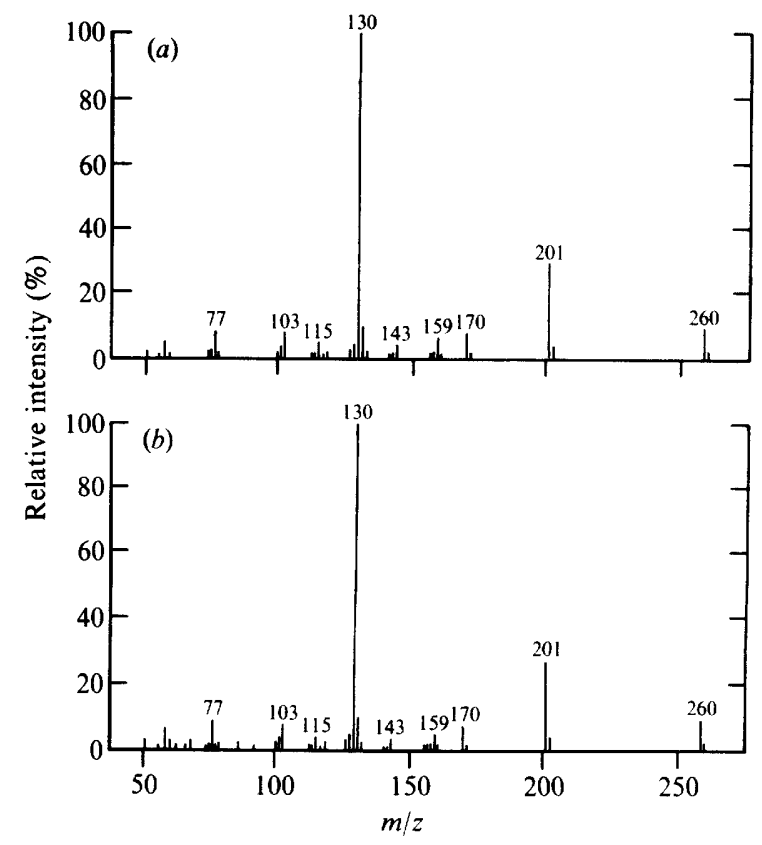

Fig. 5

Fig. 4. Electron impact ( $70 \mathrm{eV})$ mass spectra of $(a)$ authentic methyl-IAA and $(b)$ a methylated sample of putative IAA isolated from an Arthrobacter nicotianae culture grown on $100 \mu \mathrm{g} \mathrm{L}$-tryptophan $\mathrm{ml}^{-1}$. Ions characteristic of methyl-IAA are $m / z 189\left(M^{+}\right), 130$ (base peak) and 103.

Fig. 5. Electron impact $(70 \mathrm{eV})$ mass spectra of $(a)$ authentic methyl-N $\alpha$-acetyl-L-tryptophan and $(b)$ a methylated sample of putative $\mathrm{N} \alpha$-acetyl-L-tryptophan isolated from an Arthrobacter nicotianae culture grown on $200 \mu \mathrm{g} \mathrm{L-tryptophan} \mathrm{ml}^{-1}$. Ions characteristic of methyl-N $\alpha$-acetyl-L-tryptophan are $260\left(M^{+}\right), 201,170$ and 130 (base peak).

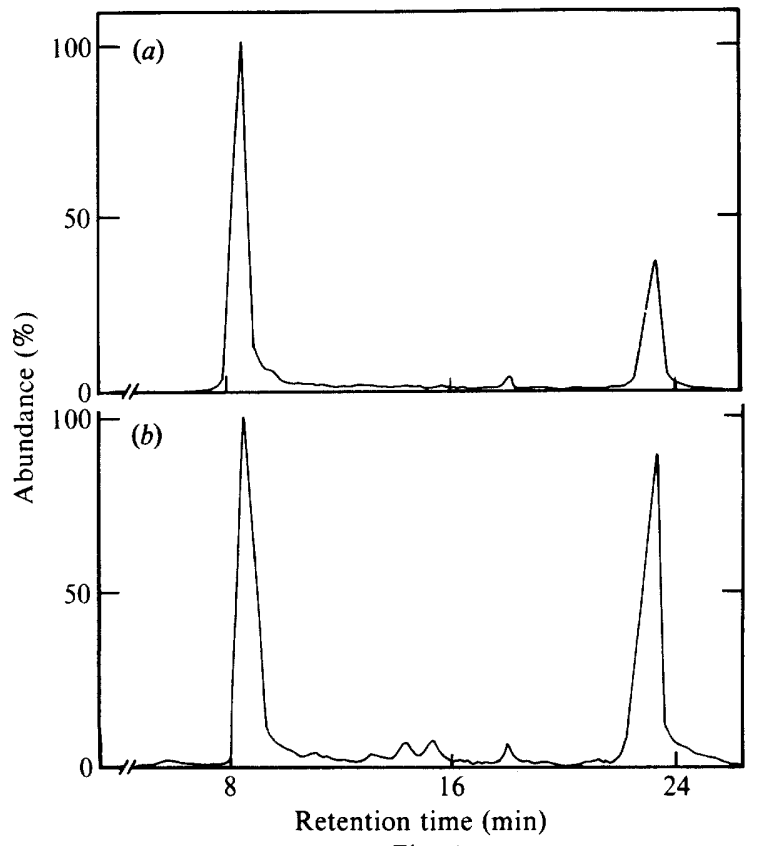

Fig. 6

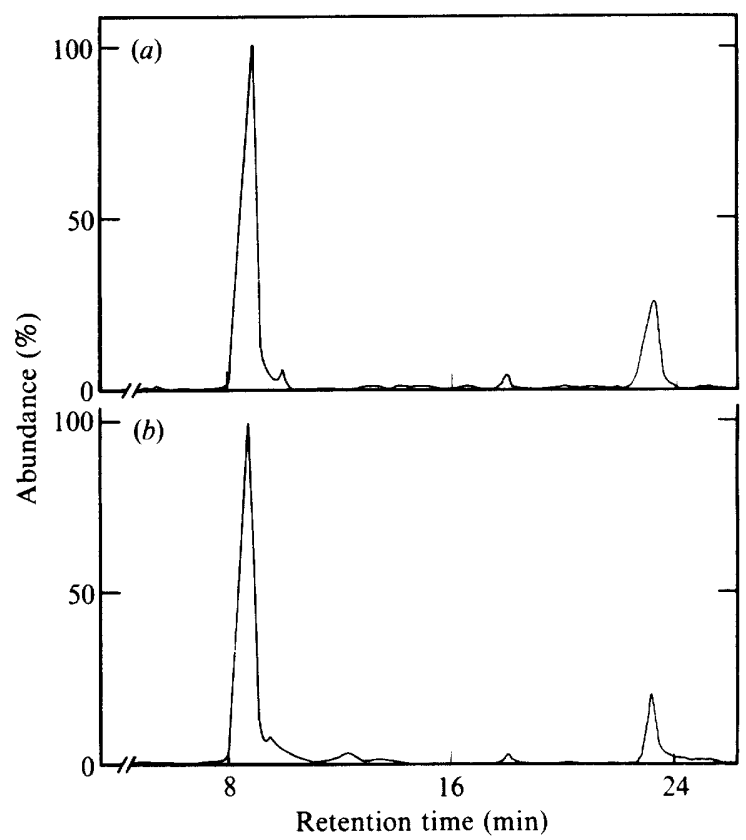

Fig. 7

Fig. 6. Relative abundance of L-tryptophan metabolites possessing the ion $m / z 130$ in cultures of Arthrobacter nicotianae grown on $100 \mu \mathrm{g}$ L-tryptophan $\mathrm{ml}^{-1}(a)$ and $600 \mu \mathrm{g} \mathrm{L-tryptophan} \mathrm{ml}^{-1}(b)$. Methyl-IAA is at $R_{t}=8.5 \mathrm{~min}$ and methyl-N $\alpha$-acetyl-L-tryptophan is at $R_{\mathrm{t}}=23.8 \mathrm{~min}$.

Fig. 7. Relative abundance of L-tryptophan metabolites possessing the ion $\mathrm{m} / \mathrm{z} 130$ in cultures of Arthrobacter globiformis grown on $100 \mu \mathrm{g} \mathrm{L}$-tryptophan $\mathrm{ml}^{-1}(a)$ and $600 \mu \mathrm{g} \mathrm{L}$-tryptophan $\mathrm{ml}^{-1}(b)$. Methyl-IAA is at $R_{\mathrm{t}}=8.5 \mathrm{~min}$ and methyl-N $\alpha$-acetyl-L-tryptophan is at $R_{\mathrm{t}}=23.8 \mathrm{~min}$. 
bolite when the cultures were grown on relatively low concentrations of L-tryptophan (up to $100 \mu \mathrm{g} \mathrm{ml}^{-1}$ ) (Figs 6 and 7). In cultures of $A$. globiformis (Fig. 7) and $A$. crystallopoietes (data not shown) formation of $\mathrm{N} \alpha$-acetylL-tryptophan was enhanced when increased L-tryptophan concentrations were supplied to the cultures. In $A$. crystallopoietes, $\mathrm{N} \alpha$-acetyl-L-tryptophan was the major metabolite when $600 \mu \mathrm{g}$ tryptophan $\mathrm{ml}^{-1}$ was supplied to the culture.

Two other minor metabolites, $\mathrm{N} \alpha$-methyl-L-tryptophan and $N, N^{\prime}$-dimethyl-L-tryptophan, were identified in most cultures (data not shown).

\section{Discussion}

The three species of Arthrobacter isolated from Azolla produced IAA in mineral medium, confirming previous observations (Forni \& Grilli Caiola, 1988).

IAA production in non-growing (i.e. M9 medium) and in actively growing (i.e. TRN medium) cultures indicated the capability of this genus to produce IAA in different growth conditions. In medium M9 Arthrobacter produced IAA only when L-tryptophan was added as substrate and showed a significant increase in the presence of high concentrations of the amino acid. The amount of IAA produced by the bacteria was within the detection limits of the Salkowski reagent (Ehmann, 1977), which is specific for IAA and does not interact with L-tryptophan or $\mathrm{N} \alpha$-acetyl-L-tryptophan.

The GC-MS analyses of the culture supernatants showed the presence of several tryptophan metabolites besides IAA, the most abundant of which was $\mathrm{N} \alpha$-acetylL-tryptophan. The production of these compounds may be a mechanism by which the bacterial cell eliminates the possible toxic effects of high concentrations of tryptophan.

The leaf cavities of Azolla imbricata and of Azolla freed of Anabaena azollae contain respectively 0.09 and 0.29 mM-tryptophan (Yun-lu et al., 1983); these tryptophan concentrations are within the range used in our experiments. It is not known what the effects of this amino acid are on A. azollae. However, Rawson (1985) demonstrated that a high concentration of tryptophan reduced nitrogenase activity and growth in Anabaena cylindrica PCC 7122. Thus in the association, A. azollae may benefit by the bacterial tryptophan utilization resulting in the synthesis of IAA.

It is likely that the bacteria excrete the hormone into the leaf cavity of the fern, where the hair cells might transfer it to the plant. The direct uptake by plants of IAA produced by bacteria has been observed in other plant-bacteria associations (Libbert \& Silhengst, 1970).
In addition, it has been demonstrated that epiphytic bacteria may increase the IAA content in plants ' Libbert et al., 1969).

The recognition that the growth and development of plants depends on growth regulators has led to the suggestion that they might benefit from the external supply of such substances. Moreover, it has been suggested that auxin may play a critical role in some symbioses, such as Nostoc-Gunnera (Towata, 1985) and in Rhizobium-legumes (Prinsen et al., 1991) Therefore it can be postulated that IAA production by the bacteria might exert a marked influence on growth and morphogenesis of Azolla. Unfortunately, in view of the contradictory reports concerning the possible action of IAA on cyanobacteria (Ahmad \& Winter, 1968; Leganes et al., 1987) it is difficult at present to predict its effect on symbiotic Anabaena azollae.

Special thanks are due to the Italian National Council of Research (CNR) for the fellowship (n.203.04.12) awarded to C. Forni for the stay in Israel. We are grateful to Mrs. Naomi Agut for excellent technical assistance.

\section{References}

Ahmad, M. R., \& Winter, A. (1968). Studies on the hormonal relationships of algae in pure culture. $I$. The effect of indole-3-acetic acid on the growth of the blue-green and green algae. Planta 78, 277286.

EhmanN, A. (1977). The Van Urk-Salkowski reagent - a sensitive and specific chromogenic reagent for silica gel thin-layer chromatographic detection and identification of indole derivatives. Journal of Chromatography 132, 267-276.

ForNi, C. \& GRIlli Caiola, M. (1988). Physiological characterization of the bacteria, isolated from the aquatic fern Azolla. In Nitrogen Fixation: Hundred Years After. Proceedings of the 7 th International Congress on Nitrogen Fixation, p. 235. Edited by H. Bothe, F. J. de Bruijn \& W. E. Newton. Stuttgart \& New York; Gustav Fisher.

Forni, C., Grilli Caiola, M. \& Gentili, S. (1989). Bacteria in the Azolla-Anabaena symbiosis. In Nitrogen Fixation with Non-Legumes. Proceedings of the 4th International Symposium on Nitrogen Fixation with Non-Legumes, pp. 83-88. Edited by F. A. Skinner, R. M. Boddey \& I. Fendrik. Dordrecht: Kluwer Academic Publishers.

Forni, C., Gentili, S., Van Hove, C. \& Grilli Caiola, M. (1990). Isolation and characterization of the bacteria living in the sporocarps of Azolla filiculoides Lam. Annali Microbiologia 40, 235-243.

Grilli Caiola, M., Forni, C. \& Castagnola, M. (1988). Bacteria in the Azolla-Anabaena association. Symbiosis 5, 185-198.

HARARI, A., KIgEL, J. \& OKon, Y. (1989). Involvement of IAA in the interaction between Azospirillum brasilense and Panicum miliaceum roots. In Nitrogen Fixation with Non-Legumes. Proceedings of the 4th International Symposium on Nitrogen Fixation with Non-Legumes, pp. 227-234. Edited by F. A. Skinner, R. M. Boddey \& I. Fendrik. Dordrecht: Kluwer Academic Publishers.

Leganes, F., Sanchez-Maeso, E. \& Fernandez-Valiente, E. (1987). Effect of indoleacetic acid on growth and dinitrogen fixation in cyanobacteria. Plant Cell Physiology 28, 529-533.

LIBBERT, E. \& SILHENGST, P. (1970). Interactions between plants and epiphytic bacteria regarding their auxin metabolism. VIII. Transfer of ${ }^{14} \mathrm{C}$-indoleacetic acid from epiphytic bacteria to corn coleoptiles. Physiologia Plantarum 23, 480-487.

LIBBERT, E., KAISER, W. \& KUNERT, R. (1969). Interactions between plants and epiphytic bacteria regarding their auxin metabolism. VI. The influence of the epyphitic bacteria on the content of extractable auxin in the plant. Physiologia Plantarum 22, 432-439. 
Nierzwicki-Bauer, S. \& AUlfinger, H. (1990). Ultrastructural characterization of eubacteria residing within the leaf cavities of symbiotic and cyanobiont-free Azolla mexicana. Current Microbiology 21, 123-129.

Peters, G. A. \& Meeks, J. C. (1989). The Azolla-Anabaena symbiosis: basic biology. Annual Review of Plant Physiology and Plant Molecular Biology 40, 193-210.

Petro, M. J. \& Gates, J. E. (1987). Distribution of Arthrobacter sp. in the leaf cavities of four species of the $\mathrm{N}$-fixing Azolla fern. Symbiosis 3, 41-48.

Plazinski, J., Taylor, R., Shaw, W., Croft, L., Rolfe, B. G. \& GuNNING, B. E. S. (1990). Isolation of Agrobacterium sp., strain from the Azolla leaf cavity. FEMS Microbiology Letters 70, 55-60.

Prinsen, E., ChauvauX, N., Schmidt, J., John, M., Wiencke, U., De Greel, J., Schell, J. \& VAN ONCKelen, H. (1991). Stimulation of indole-3-acetic acid production in Rhizobium by flavonoids. FEBS Letters 282, 53-55.

Rawson, D. M. (1985). The effects of exogenous amino acids on growth and nitrogenase activity in the cyanobacterium Anabaena cylindrica PCC 7122. Journal of General Microbiology 131, 2549-2554.
Sagee, O., Maoz, A., Mertens, R., Goren, R. \& Riov, J. (1986). Comparison of different enzyme immunoassays for measuring indole-3-acetic acid in vegetative citrus tissue. Physiologia Plantarum 68, 265-270.

SchlenK, H. \& Gellerman, J. L. (1960). Esterification of fatty acids with diazomethane on a small scale. Analytical Chemistry 32, 14121414.

TowatA, E. M. (1985). Morphometric and cytochemical ultrastructural analyses of the Gunnera kaalensis/Nostoc symbiosis. Botanical Gazette 146, 293-301.

Wallace, W. H. \& Gates, J. E. (1986). Identification of eubacteria isolated from leaf cavities of four species of the $\mathrm{N}$-fixing Azolla fern as Arthrobacter Conn and Dimmick. Applied and Environmental Microbiology 52, 425-429.

Yun-lu, X., Ke-Zhi, B., Sai-ling, Y., Cheng, C., Bao-zhen, H. \& XIU-ZHANG, H. (1983). Nitrogenous compounds of the leaf cavity liquid of Azolla in relation to the symbiosis of Azolla and Anabaena azollae. Acta Botanica Sinica 25, 82-86. 